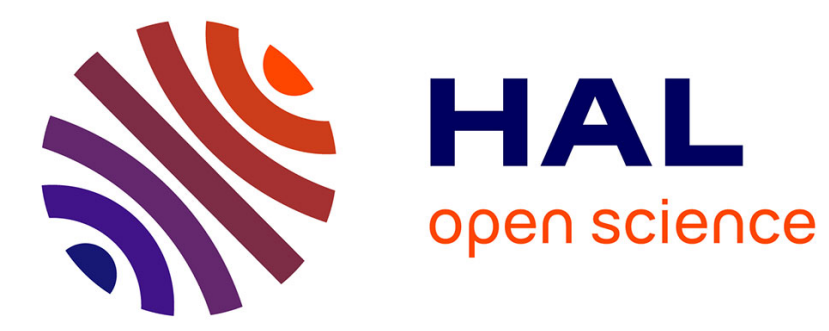

\title{
RECHERCHES SUR LE PROCÉDÉ BURRI DE NUMÉRATION BACTÉRIENNE PAR STRIES (suite)
}

\author{
W. Dorner, P. Demont
}

\section{To cite this version:}

W. Dorner, P. Demont. RECHERCHES SUR LE PROCÉDÉ BURRI DE NUMÉRATION BACTÉRIENNE PAR STRIES (suite). Le Lait, 1931, 11 (110), pp.1005-1021. hal-00895034

\section{HAL Id: hal-00895034 \\ https://hal.science/hal-00895034}

Submitted on 1 Jan 1931

HAL is a multi-disciplinary open access archive for the deposit and dissemination of scientific research documents, whether they are published or not. The documents may come from teaching and research institutions in France or abroad, or from public or private research centers.
L'archive ouverte pluridisciplinaire HAL, est destinée au dépôt et à la diffusion de documents scientifiques de niveau recherche, publiés ou non, émanant des établissements d'enseignement et de recherche français ou étrangers, des laboratoires publics ou privés. 


\section{LE LAIT}

REVUE GÉNERALE DES QUESTIONS LAITIERES

\section{S O M M A I R E}

Mémoires originaux :

W. Dorner et P. Demont. Recherches sur le procédé Burri de numération bactérienne par stries (fin) . . . 1005

L. Musso et Germain. - Variations de la matière grasse du lait de vache dans la région d'Alger (à suivre) . . 1022

Jean Piwn. - Sur la teneur en fer des caś́ines industrielles 1038

Bibliographie analytique :

10 Livres . Rournaux, Revues, Sociétés 1055

savantes........ 1057

$3^{\circ}$ Brevets............. 1077

\section{Bulletin bibliographique :}

$1^{0}$ Les Livres. $2^{\circ}$ Journaux, Revues, Sociétés savantes...... 1080

$3^{\circ}$ Brevets . . . . . . . . . 1085

Documents et informations :

Les àssociations coopératives de vente aux Etats-Unis ‥ 1086

Exportation de beurre russe . . 1087

Union centrale des coopératives laiteries lettones .... 1088

L'allaitement maternel en Rou. manie . . . . . . 1088

Production du lait au Danemark . . . . . . . 1088

Correspondance . . . . 1089

Table des matières . . . . . 1090

Table des auteurs. . . . . 1102

Table des ouvrages analysés . 1122

\section{VÉMOIRES ORIGINAUX (1)}

\section{RECHERCHES SUR LE PROCÉDÉ BURRI DE NUMÉRATION BACTÉRIENNE PAR STRIES}

Par W. DORNER et P. DEMONT, de l'Établissement fédéral d'industrie laitière et de bactériologie, Liebefeld-Berne.

Directeur : Prof. Dr. R. BURRI.

(suite)

VI. PEGRÉ DE SÉCURITÉ ET DOMAINE D'UTILISATION DU PROCÉDÉ DE NUMÉRATION DE BURRI.

Il ne suffit pas qu'un procédé soit simple, rapide et peu coûteux pour être satisfaisant. Il faut, avant tout, qu'il soit sûr et qu'il donne des résultats concordants. C'est ce point que nous allons examiner dans le chapitre qui suit.

a) MOYEN DE DETERMINER LA VALEUR D'UN PROCÉE DE NUMERATION BACtÉrienNe. - Trois points principaux doivent être pris en

(1) Reproduction interdite sans indication de source. 
considération lors de l'examen de procédés destinés à la détermination d'une dimension ou valeur quelconque. Ce sont :

10 la dispersion lors de déterminations parallèles ;

20 la concordance des résultats obtenus avec ceux d'autres procédés, et

$3^{\circ}$ le domaine d'utilisation du procédé à l'essai.

Tous les procédés de numération bactérienne présentent des sources d'erreur relativement grandes ; aucun d'entre-eux ne peut être considéré comme procédé-étalon et servir de mesure pour la valeur des autres. De ce fait, la concordance avec d'autres procédés n'a pas un poids très grand en tant que les différences ne sont pas inexplicables. Il en est autrement de la dispersion lors de détermination parallèles. Pour qu'un procédé soit satisfaisant, il faut que les résultats de détermination parallèles concordent aussi bien que possible, ou qu'en d'autres mots, leur dispersion soit faible.

Les bactériologistes américains ont voué une somme de travail considérable à l'étude des procédés de numération bactérienne. Ils ont, entre autres, déterminé soigneusement ces facteurs qui influencent la dispersion. C'est ainsi qu'on a découvert en particulierle fait que plus le nombre des colonies que l'on compte sur le milieu dẻ culture est élevé, plus, toutes proportions gardées, la dispersion lors de déterminations parallèles, est réduite. Il s'agit là, d'une règle générale d'où découle qu'il existe dans la numération des colonies un nombre limite au-dessous duquel les résultats sont incertains ; il en existe également un en dessus. Indépendamment du fait qu'il est impossible, pour des raisons pratiques, de compter plus d'un certain nombre de colonies, il faut tenir compte du phénomène d'inhibition mutuelle des bactéries lorsqu'elles se trouvent trop près les unes des autres, et qu'en plus, des colonies trop rapprochées peuvent confluer. Dans les deux cas, on trouvera un nombre de colonies inférieur à ce qu'il devrait être. Il se peut alors que la dispersion des résultats de déterminations parallèles soit très faible. Les résultats n'en seront pas moins faux, puisque les moyennes seront trop basses.

Bien qu'il existe des limites aux nombres des colonies en dehors desquels les résultats ne peuvent plus être considérés comme sûrs, le domaine d'utilisation des procédés culturaux de.numération bactérienne est illimité vers le haut, car la méthode de dilution déjà utilisée par Pasteur permet de réduire à volonté le nombre de colonies qui doivent se développer sur les milieux. En sens contraire, la limite d'utilisation est fixée par la quantité maximale de liquide qu'il est possible d'ensemencer sur le milieu. Cette quantité peut être fixée à $0 \mathrm{~cm}^{3} 01$ (10 anses) pour le procédé Burri et à 1 ou $2 \mathrm{~cm}^{3}$ pour le procédé des plaques.

$\mathrm{Vu}$ l'importance que présente la dispersion pour apprécier les 
procédés de numération bactérienne, une question se pose: à savoir, en quels termes l'exprimer. On peut, en prenant, tout d'abord le procédé le plus primitif, indiquer la différence entre les variantes extrêmes. Ce procédé ne donne cependant pas une image fidèle de la dispersion, parce que, d'une part, selon les calculs de probabilité, celle-ci sera d'autant plus grande que le nombre des déterminations sera grand, et parce que, d'autre part, le grand nombre des résultats touchant de près à la moyenne n'est pas pris en considération. En outre, la dispersion exprimée en chiffres absolus augmente avec la moyenne. Il est donc nécessaire d'exprimer la dispersion en tenant compte des résultats se rapprochant de la moyenne et d'une manière indépendante de la grandeur de la moyenne. BREED (8) a recommandé pour apprécier le procédé des plaques d'exprimer la dispersion relative en indiquant combien pourcent des résultats de déterminations parallèles tombaient entre $20 \%$ en plus et $20 \%$ en moins de la moyenne. Il fixe la moyenne quelque peu arbitrairement en négligeant les variantes extrêmes. Ce procédé est utilisable. Il constitue toutefois exactement le contraire du procédé mentionné en premier lieu, parce qu'il néglige entièrement les variantes extrêmes. C'est un inconvénient, car ce sont justement les résultats extrêmes qui peuvent discréditer un procédé.

C'est pour cette raison que nous nous sommes décidés à employer la méthode des moindres carrés pour ealculer et exprimer la dispersion des résultats de nos expériences. Cette méthode tient compte aussi bien des variantes extrêmes que des variantes moyennes et donne de ce fait une image réelle de la dispersion. Nous nous permettons à cet endroit de remercier M. le $\mathrm{D}^{\mathrm{r}} \mathrm{S}$. BLuMER, à Berne, pour les indications et les conseils qu'il nous a prodigués pour l'emploi du calcul des variations. Qu'il nous soit encore permis de faire quelques observations au sujet d'un travail de MUDGE et LAwLER (9), qui arrivent à la conclusion surprenante que la méthode des moindres carrés ne se prête pas à l'appréciation des procédés de numération bactérienne. Dans ses grandes lignes, le raisonnement qui amène ces auteurs à cette conclusion est le suivant : la numération bactérienne sur plaques a rendu d'immenses services dans l'amélioration de la qualité du lait. La méthode des moindres carrés démontre que ce procédé est entâché d'erreurs considérables. Par conséquent, la méthode des moindres carrés est à rejeter. Ce raisonnement est plutôt curieux. A notre avis, il ne sert de rien de vouloir fermer les yeux sur l'inexactitude que présentent tous les procédés de numération bactérienne en condamnant une méthode mathématique. Au contraire, il est important que celui qui doit interprêter des résultats de numérations bactériennes soit bien renseigné sur la valeur des chiffres qu'il a sous les yeux. Comme partisans convaincus de la numération bactérienne pour déterminer la qualité du lait, nous ne eraignons pas de dire qu'une erreur de 20,30 ou même $50 \%$ dans la teneur en germes est sans aucune importance. 
b) BUt des eXPÉriences leur organisation. - Le but des expériences décrites dans la suite était de se faire une image exacte des points soulevés plus haut : la dispersion des résultats et le domaine d'utilisation. Le troisième point : concordance des résultats du procédé Burri avec le procédé des plaques a été examiné dans un chapitre spécial. En principe, nous avons cherché dans nos expériences à faire un grand nombre de déterminations parallèles, tant par le procédé de Burri que par celui des plaques, en maintenant égales toutes les conditions qui affectent le développement microbien. Nous avons commencé nos expériences avec du lait. Plus tard, nous avons préféré l'emploi de cultures pures de str. lactis. La teneur microbienne du lait est en effet assez variable ; aussi, lorsqu'il s'agit de faire des sérjes de 200 déterminations parallèles il est important d'être fixé d'avance sur la teneur en bactéries afin de ne pas être obligé de faire plusieurs dilutions. La préparation d'une seule dilution double en effet tout le travail. Au début, nous avions des séries d'environ 200 déterminations parallèles. Plus tard, nous avons réduit ce nombre à 25-35 par série. Pour avoir cependant un certain contrôle, 7 à 10 de ces séries plus petites étaient conduites parallèlement, en ensemençant chacune d'elles avec un nombre de bactéries qui est multiple de celui de la série précédente. L'emploi de dilutions variées du même liquide d'ensemencement a permi d'étudier avec plus de facilité l'influence du nombre de colonies sur la dispersion. Cette manière de faire nous a permi également d'obtenir des données précieuses sur le domaine d'utilisation du procédé Burri.

c) Technique des essais. - Nous donmons ci-après une description de la manière dont nous avons effectué les essais.

1. Milieux de culture. - Dans chaque série nous avons utilisé pour les plaques et pour les tubes de la gélose de même origine. La gélose en tubes traitée comme cela est décrite au chapitre III. La gélose pour les plaques a été stérilisée en ballons à raison de $300 \mathrm{~cm}^{3}$ par ballon.

La stérilisation des tubes et des ballons a été effectuée en une seule fois dans le même autoclave. Tout a été mis en œuvre pour que le milieu de culture fut exactement le même dans les plaques et dans les tubes, sauf en ce qui concerne naturellement les différences inhérentes aux deux procédés.

2. Préparation des plaques. - Pour préparer les plaques en un minimum de temps, tout le personnel du laboratoire a été mobilisé. Nous sommes arrivés ainsi à en préparer plus de 200 en 50 minutes. Au début, $1 \mathrm{~cm}^{3}$ d'un échantillon de lait bien mélangé a été ajouté à 1 litre d'eau stérilisée et maintenue à 5-6 ${ }^{\circ} \mathrm{C}$. Après agitation, $1 \mathrm{~cm}^{3}$ de cette dilution était mis dans chaque boîte de Petri à l'aide d'une 
pipette. Une autre personne mettait environ $10 \mathrm{~cm}^{3}$ de gélose fondue et refroidie à $45^{\circ}$ dans chaque boîte de Petri. Une troisième personne mélangeait soigneusement la gélose au liquide d'ensemencement et une quatrième numérotait et serrait les plaques. Il a été procédé exactement de la même manière pour les séries faites aveo des eultures pures de str. lactis, avec la différence qu'au lieu de lait, nous avons employé une dilution appropriée de culture en bouillon de viande de cheval, âgée de 6 à 8 jours.

3. Ensemencement des tubes de gélose inclinée. - Pendant la préparation des plaques, l'un de nous procédait à l'ensemencement des tubes de gélose. L'anse employée à cet effet au début n'avait pas la grandeur normale, son diamètre n'étant que de $1 \mathrm{~mm}$. 22. De ce fait, les résultats des esais des séries 1-6 ne peuvent pas être utilisés pour comparer d'une manière absolue les résultats des plaques avec ceux des tubes. En ce qui concerne la dispersion et le domaine d'utilisation du procédé Burri, ce fait n'enlève rien à la valeur de nos résultats. L'ensemencement a eu lieu à partir de lait non dilué qui a servi pour les plaques ou de la même culture de str. lactis diluée mille fois au moins. Ces liquides étaient maintenus également à $5-6^{\circ} \mathrm{C}$. pendant l'ensemencement et agités régulièrement après a voir ensemencé 10 tubes. L'ensemencement lui-même a eu lieu comme cela est décrit au chapitre III ; seulement comme nous avions toujours le même liquide d'ensemencement, l'anse n'a pas été flambée entre chaque tube. Il nous fallait dans la règle 45 minutes pour ensemencer 250 tubes.

4. Incubation et dénombrement des colonies. - Immédiatement après l'ensemencement les plaques et les tubes étaient portés dans une chambre-étuve assez vaste maintenue à $30^{\circ} \mathrm{C}$. On prenait la précaution de ne jamais mettre beaucoup de plaques les unes sur les autres et l'on faisait en sorte que l'air puisse circuler librement entre les piles.

Le dénombrement des colonies développées avait lieu après 48 heures d'incubation. A cet effet, tout le personnel du laboratoire était mobilisé à nouveau. Ce dénombrement se faisait dans la règle à l'œil nu. La loupe n'a été employée qu'en cas de doute. Pour les deux premières séries, le dénombrement fut répété après 72 heures d'incubation.

5. Mise en valeur des résultats. - Les nombres de colonies obtenues ont été mis en valeur selon les indications de JOHANNSEN (10). Comme, a notre avis, le procédé de JoHANNSEN est trop peu connu, nous donnerons le détail des calculs pour l'une des séries et nous choisissons à titre d'exemple l'une des plus petites.

Avant de procéder, il est nécessaire de définir quelques termes qui seront employés plus loin. L'erreur moyenne, $\mu$, est en termes absolus la mesure pour la dispersion. Elle est donnée par la formule ordinaire suivante : 


$$
\psi= \pm \sqrt{\frac{\Sigma}{n}}
$$

En appliquant cette formule, le calcul est très long ; c'est pourquoi nous avons préféré employer le procédé simplifié indiqué par JoHANNSEN. L'erreur moyenne, $\mu$, est égale à la déviation standard $\sigma$. Pour permettre la comparaison de séries à moyennes différentes quant à l'ordre de grandeur, on a exprimé l'erreur moyenne en \% de la moyenne arithmétique. C'est ce qu'on nomme le coefficient de variation. Ce coefficient très important exprime l'indice de sécurité d'un procédé.

\section{CALCUL DE L'ERREUR MOYENNE ET DU CCEFFICIENT DE VARIATION.}

Données de la $4^{\mathrm{e}}$ série, dilution 4 , tubes :

Les 37 tubes ensemencés ont donné les résultats suivants au dénombrement des colonies :

$47,74,79,99,62,63,66,78,100,85,103,87,84,68,63,83,83,77$, $75,63,86,70,73,68,80,78,90,71,77,90,80,92,70,73,95,59,63$.

La première opération est de répartir ces résultats par classes de valeur. Nous avons fixé en général les classes à environ $10 \%$ de la moyenne arithmétique du nombre de colonies. Dans le cas particulier, chaque classe embrasse un intervalle a de 8 unités.

$\begin{array}{lrlllllllll}\text { Classes : } 46 & -54 & -62 & -70 & -78 & -86 & -94 & -102 & -110 \\ \text { nombre de } & & 1 & 8 & 8 & 11 & 4 & 3 & 1 \\ \text { résultats: } & 1 \quad \begin{array}{c}1 \\ \text { total }=\mathrm{n}=37\end{array} & & & & & \end{array}$

L'opération suivante consiste à choisir arbitrairement un point de départ $\mathrm{A}$ pour le calcul de la moyenne arithmétique ; dans la règle, on le choisit dans la classe qui contient le plus de résultats. Ici nous avons choisi 82 , le centre de la classe 78-86. On numérote ensuite les classes à partir de ce point en classes positives et classes négatives. On a alors :

$\begin{array}{rrrrrrrrr}\text { classes } & -4 & -3 & -2 & -1 & 0 & +1 & +2 & +3 \\ \text { nombre de variantes } & 1 & 1 & 8 & 8 & 11 & 4 & 3 & 1\end{array}$

Pour le calcul de la moyenne, on fait d'abord la différence entre le nombre des variantes des classes de même rang que l'on multiplie par le numéro de leur classe. On a alors :

$$
\begin{array}{rlrl}
11 \times 0 & =0 & \\
+4 & -8 \times 1 & =-4 & \\
+3 & -8 \times 2 & =-10 & \mathrm{~b}=\frac{-18 \times 8}{37}=-3.88 \\
+1 & -1 \times 3 & =0 & \mathrm{~A}=82 \\
-1 \times 4 & =-4 &
\end{array}
$$


Ce total doit être multiplié par la valeur d'une classe et divisé par 'le nombre total des variantes. Pour obtenir la moyenne M, le résultat b est ensuite, suivant sa signification, additionné ou soustrait du point de départ $\mathrm{A}$. Dans le cas présent, on $\mathrm{a}: \mathrm{M}=\mathrm{A}+\mathrm{b}=82-3.88$ $=78,12$.

Cette manière de calculer la moyenne arithmétique peut paraître compliquée. Elle simplifie cependant énormément le calcul de l'erreur moyenne, pour laquelle elle donne tous les éléments, On verra dans la suite, combien plus simple est le calcul fait au moyen des données ci-dessus que lorsqu'il faut prendre chaque résultat séparément et en calculer la déviation. L'erreur moyenne $\mu$ s'obtient par la formule suivante :

$$
\mu=\sqrt{\frac{\Sigma p a^{2}}{n}-b^{2}}
$$

Pratiquement, voilà comment on procède :

On reprend le tableau de répartition en classes :

$$
\begin{array}{rrrrrrrr}
-4 & -3 & -2 & -1 & 0 & -1 & -2 & -3 \\
1 & 1 & 8 & 8 & 11 & 4 & 3 & 1
\end{array}
$$

on additionne le nombre des variantes des classes de même rang, on les,multiplie par,le carré du numéro de leur classe et l'on additionne les résultats. On a alors :

$$
\begin{array}{r}
11 \times 0=0 \\
8+4=12 \times 1=12 \\
8+3=11 \times 4=44 \\
1+1=2 \times 9=18 \\
1+0=1 \times 16=16 \\
\text { Total }=\frac{90}{\frac{90 \times 64}{37}}-2,88^{2}=\mu=12.1
\end{array} \quad \text { Le total est à multiplier par } a^{2},
$$

la valeur d'une classe étant $a=8$, le résultat est à diviser par le nombre $\mathrm{n}$ de variantes, soit 37 . On soustrait encore $\mathrm{b}^{2}, \mathrm{~b}$ étant égal à 3.88 et l'on extrait la racine carrée pour obtenir l'erreur moyenne.

Le coefficient de variation $v$ est donné par la formule :

$$
\mathrm{v}=\frac{100 \mu}{\mathrm{M}} \text { soit } \frac{100 \times 12,1}{78,12}=15,4
$$

Pour des raisons pratiques, il est impossible de donner ici les résultats au complet. Cette suite de plus de 3.500 résultats de numérations bactériennes serait d'une lecture par trop fastidieuse. Nous avons rassemblé les résultats de toutes les séries d'essais dans le tableau VII.

Voyons maintenant quels sont les enseignements qui en découlent. Tout d'abord, malgré le diamètre trop petit de l'anse dans les séries 1-6, on y trouve partout, lorsqu'on ensemence du lait, un nombre plus élevé de colonies sur gélose penchée que sur plaques. Lorsqu’on 
TABLEAU VII

Résultats de Numérations BAGTérienNes Parallèles SUR PLAQUeS ET SELON BURRI.

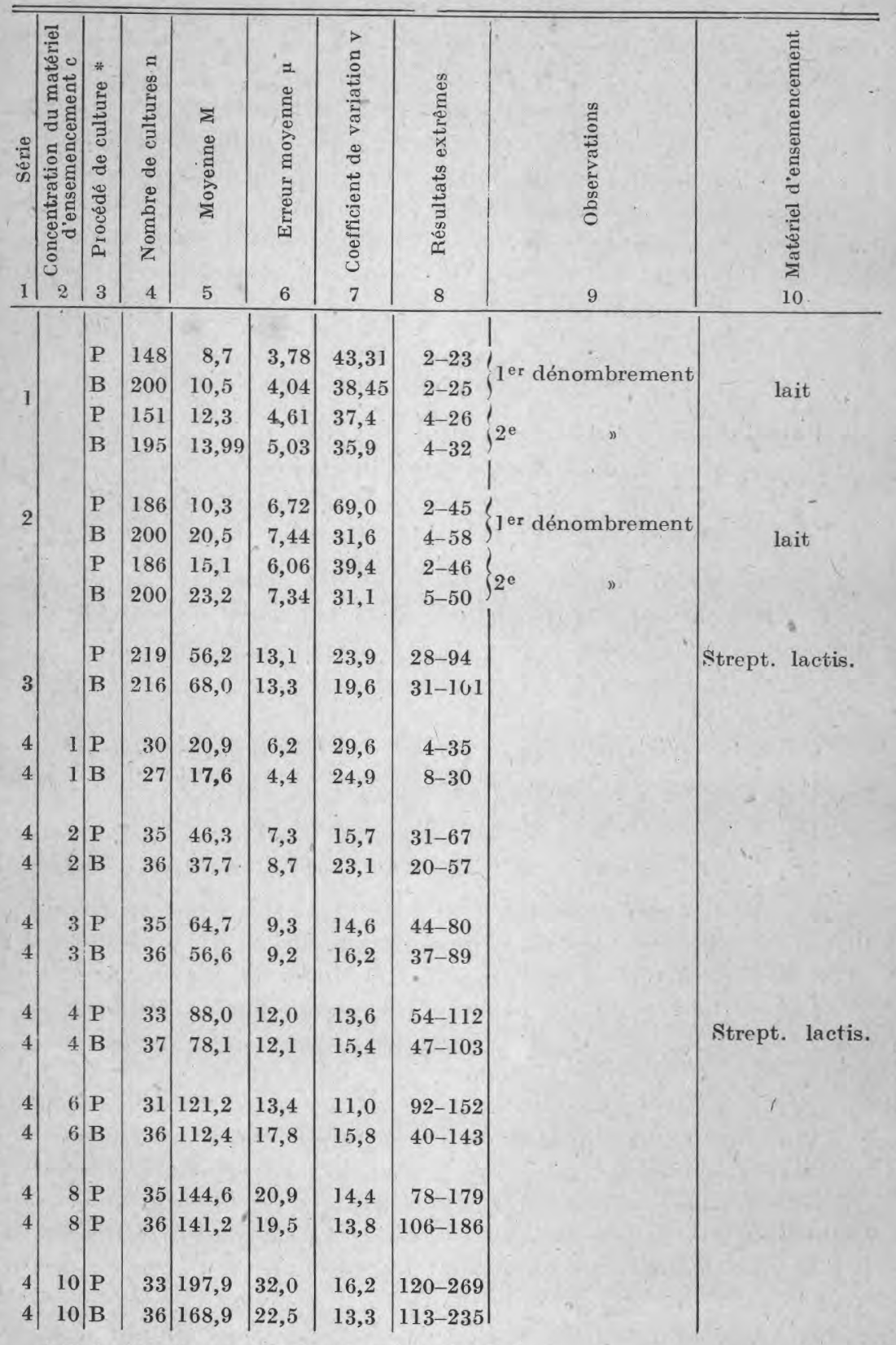

* P signifie plaques et B signifje stries selon Burri. 
TABLEAU VII (suite)

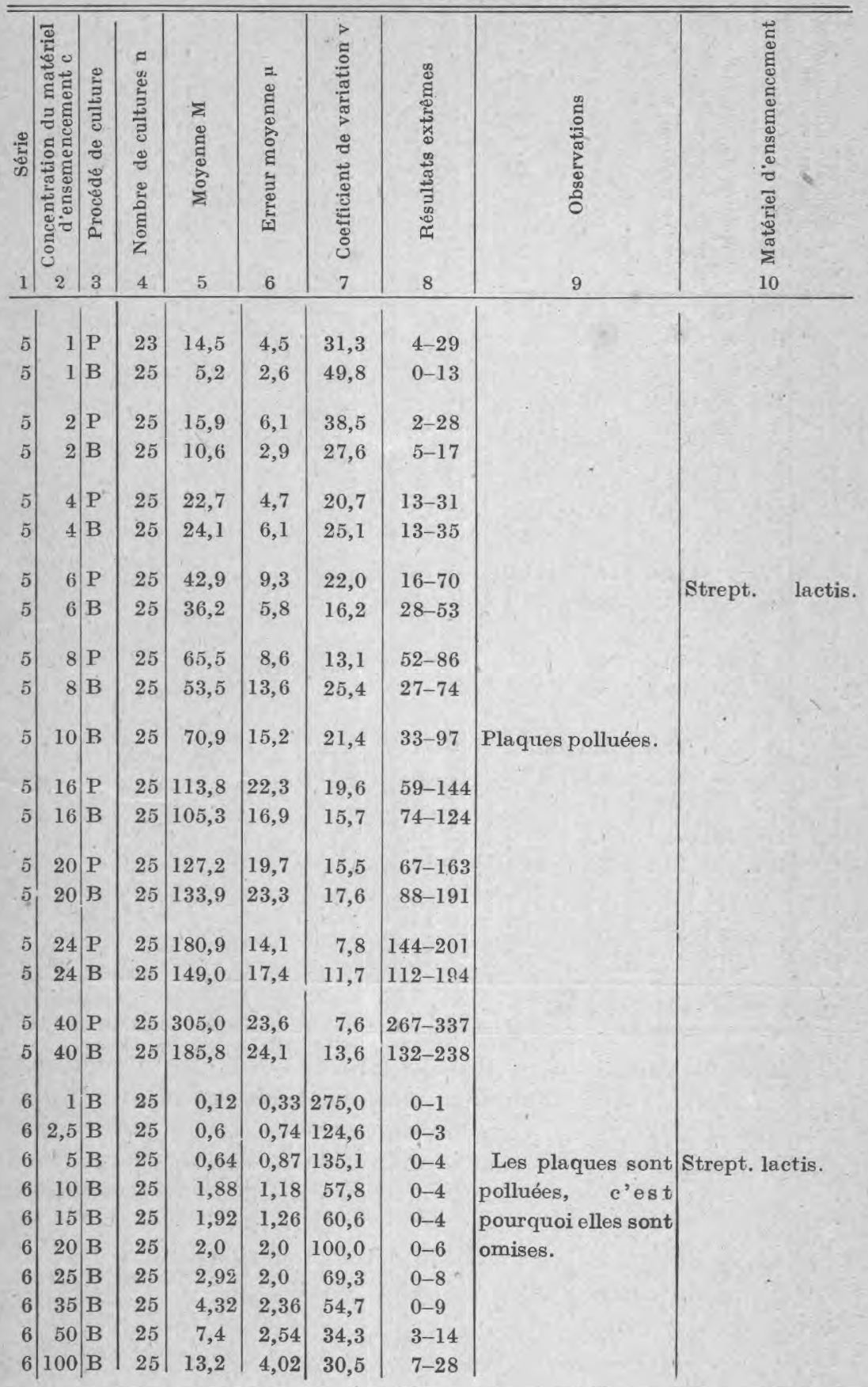


TABLEAU VII (suite)

\begin{tabular}{|c|c|c|c|c|c|c|c|c|c|}
\hline ॠ & 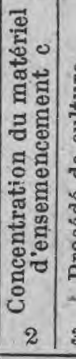 & 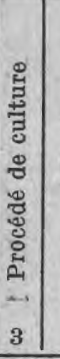 & 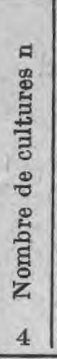 & 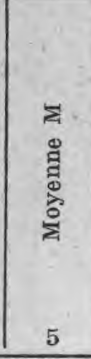 & 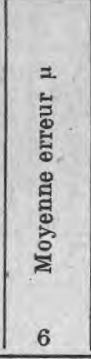 & 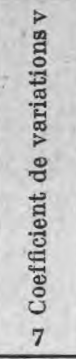 & 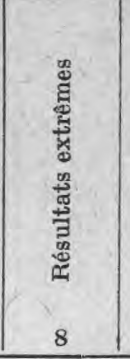 & 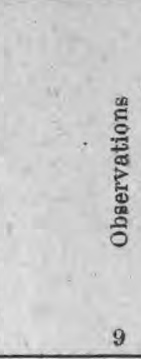 & 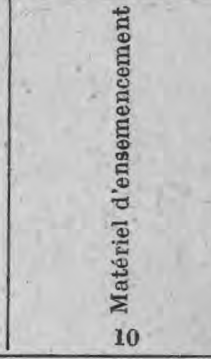 \\
\hline 7 & & $P$ & 23 & 74,2 & 12,3 & 16,6 & 51-118 & & \multirow[t]{2}{*}{ Lait homogén. } \\
\hline 7 & & B & 30 & 64,6 & 11,9 & 18,4 & $43-82$ & & \\
\hline 8 & & $\mathbf{P}$ & 25 & 121,9 & 9,25 & 7,6 & $\mid 106-135$ & & \multirow{2}{*}{ ") } \\
\hline 8 & & B & 29 & 90,1 & 15,0 & 16,6 & $62-128$ & & \\
\hline 9 & & $\mathrm{P}$ & 25 & 38,5 & 4,6 & 11,9 & $26-47$ & & \multirow[b]{2}{*}{ " } \\
\hline 9 & & B & 25 & 47,1 & 9,0 & 19,5 & $33-62$ & & \\
\hline 10 & & $\mathbf{P}$ & 25 & 123,0 & 14,7 & 11,9 & $102-167$ & & \\
\hline 10 & & B & 30 & 203,3 & 26,5 & 12,1 & $155-258$ & & \\
\hline 11 & & $\mathbf{P}$ & 25 & 69,3 & 6,2 & 9,0 &, $58-84$ & & \multirow{2}{*}{ D) $\quad$ (1) } \\
\hline 11 & & B & 30 & 162,3 & 18,9 & 11,7 & $122-195$ & & \\
\hline 12 & & $\mathrm{P}$ & 25 & 12,9 & 3,0 & 23,2 & $9-21$ & & \multirow{2}{*}{$" \quad$ " } \\
\hline 12 & & B & 30 & 24,5 & 4,26 & 17,3 & $17-35$ & & \\
\hline 13 & & $P$ & 25 & 5,5 & 2,6 & 47,2 & $2-10$ & & \multirow{2}{*}{ " $\quad$ " } \\
\hline 13 & B & B & 30 & 10,2 & 3,95 & 38,7 & $3-19$ & & \\
\hline 14 & $\mathbf{P}$ & $P$ & 25 & 12,5 & 2,4 & 19,3 & $9-18$ & & \multirow{2}{*}{ Lait ordinaire. } \\
\hline $14 \mid$ & B & B & 30 & 51,8 & 19,5 & 37,8 & $25-84$ & & \\
\hline
\end{tabular}

Total $: \mathrm{B}=1.989 . \quad$ Total $: \mathrm{P}=1.543$.

ensemence des cultures pures diluées dans de l'eau, c'est le contraire qui est la règle. L'explication de ce phénomène réside dans le fait que l'anse que nous avons employée avait un diamètre trop petit. C'est une confirmation de ce que nous avons avancé lors de la discussion de la grandeur de l'anse et de son emploi pour ensemencer des dilutions de lait.

L'indice de sécurité des procédés de numération bactérienne, tel qu'il ressort du coefficient de variation, est des plus intéressants. II découle, en effet, du tableau, que le coefficient de variation est en général d'autant plus grand que le nombre des colonies dénombrées 
est petit. Comme il semblait y avoir là une relation intéressante, nous avons essayé de rechercher si, en représentantla chose graphiquement, il y avait moyen d'arriver à un résultat plus clair et plus précis, C'est ce que montre le graphique de la fig. 2 , qui comprend les résultats des séries 1 à 6 .

Fig. 2

RELATION ENTRE LE COFFICIENT DE VARIATION ET LE NOMBRE MOYEN DE GOLONIES SUR PLAQUES ET SUR AGAR PENCHÊ (I)

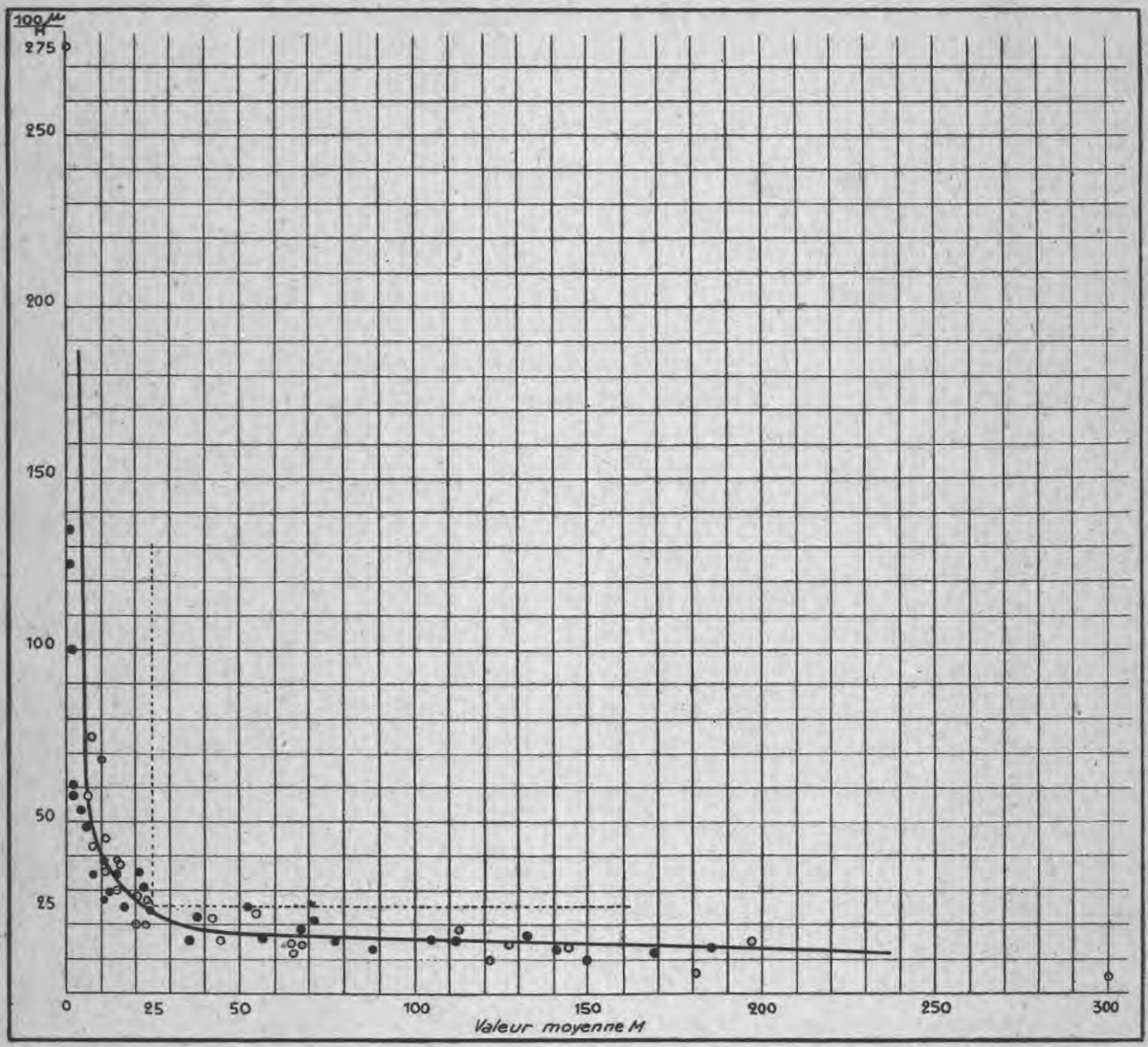

Chaque eercle représente le résultat d'une série de numérations sur plaques, tandis que les points reprêsentent les résultats des séries selon Burri. Les nombres moyens sont portés en abcisses et les cefficients de variation en ordonnées.

Iิ Un fait du plus haut intérêt se dégage du graphique de la fig. 2. A part la confirmation de la loi générale énoncée plus haut, il en découle qu'au-dessous d'un certain nombre moyen de colonies la dispersion des résultats augmente très rapidement tandis qu'au-dessus elle diminue lentement. La courbe obtenue en portant les nombres moyens

(1) Le rond de l'ordonnée 275 doit être plein. 
de colonies en abscisses et les coefficients de variation en ordonnées a, en effet, la forme d'une hyperbole. Cette observation permet de fixer sans tarder un point très important : le nombre minimum de colonies qu'il faut avoir pour obtenir un résultat relativement exact. Nous savons, en effet, qu'en théorie, plus le nombre des colonies est grand, plus petite est la dispersion relative des résultats. D'autre part, il est clair que, pour des raisons d'ordre pratique, on ne peut compter un nombre illimité de colonies. Il faut donc fixer une limite et aussi basse que possible. Etant donné que l'hyperbole présente sa plus forte courbure à environ 25 , nous pensons devoir fixer le nombre minimum de colonies à ce chiffre - Il est intéressant de relever que les bactériologistes américains ont fixé à 30 la limite inférieure du nombre de colonies pour le dénombrement des bactéries sur plaques. Etant donné qu'ils sont arrivés à ce résultat par d'autres voies, on conviendra qu'it s'agit de quelque chose de plus que d'une simple coïncidence si nous arrivons au chiffre de 25 .

Une fois le minimum de colonies fixé, on peut se prononcer plus exactement sur la précision des résultats de numération bactérienne. En reprenant le graphique, on voit que le nombre 25 de colonies correspond au coefficient de variation 25. Cela signifie que dans la règle, les résultats de numération bactérienne, se basant sur le dénombrement d'au moins 25 colonies, seront entachées d'une erreur moyenne de $\pm 25 \%$ au maximum. Normalement, cette erreur sera de $15-20 \%$. En effet, le coefficient de variation indique la variation normale d'un résultat isolé. Lorsqu'il s'agit d'une moyenne de 2 ou de 3 résultats, l'erreur probable de $25 \%$ tombe à 17 et à $14 \%$. Il y a encore un point à élucider, celui de savoir s'il faut s'en tenir strictement à la limite indiquée de 25 colonies ou, si des résultats se basant sur un plus petit nombre peuvent encore avoir quelque valeur. Nous pensons que, spécialement lorsqu'on examine du lait très pauvre en bactéries, des résultats se basant sur moins de 25 colonies ont malgré tout leur valeur. Supposons, par exemple, un lait qui donne 5 colonies sur un tube ensemencé d'une anse. Dans ce cas, il faut compter peut-être avec une erreur moyenne de $\pm 50 \%$. La teneur réelle en bactéries peut done être entre 2.500 et 7.500. Malgré cette erreur très grande, ce résultat signifie quelque chose. Il signifie que l'échantillon de lait examiné est très pauvre en bactéries. Par contre, on ne saurait prétendre qu'il pût exister une différence significative entre un lait donnant 4 et un lait donnant 8 colonies. Il conviendra de tenir compte de ce fait lors de la fixation de normes pour le paiement du lait selon sa teneur en bactéries. Si, par exemple, on se mettait d'accord pour fixer à 10.000 et moins le nombre de bactéries qu'un lait peut contenir pour obtenir une prime, il y aurait lieu d'exiger $10.000 \pm 50 \%$. En d'autres termes, il faudrait des teneurs en bactéries allant jusqu'à 15.000 . 
Le graphique peut nous enseigner encore autre chose. Nous l'avons considéré jusqu'ici dans son ensemble, sans faire de différence entre les points et les cercles qui représentent respectivement les résultats des numérations sur plaques et sur gélose penchée. L'examen du graphique en considérant séparément les points et les cercles révèle que les uns et les autres se comportent de la même manière. Ils sont tellement entremêlés qu'il est bien difficile de voir une différence entre plaques et tubes. Il semble toutefois que les plaques ont un léger avantage sur les tubes. Cet avantage est si peu marqué, qu'on peut conclure qu'à nombre égal de colonies, la dispersion sur plaques correspond à très peu de chose près à la dispersion sur tubes ensemencés avec l'anse. Etant donné, d'autre part, que le procédé de l'anse donne naissance, en général, à un nombre de colonies bien supérieur à celui des plaques, il se trouve que, comparée de cette manière, la dispersion des résultats de la numération bactérienne selon BuRRI est plutôt inférieure à celle du procédé des plaques. Ce point est important. Nous en concluons : Pour la concordance des résultats entre eux, le procédé Burri est l'égal du procédé classique des plaques. Cette conclusion est quelque peu en contradiction avec les résultats publiés par RICHTER (1. c.). Cet auteur arrive, en effet, à la conclusion que le procédé de BuRRI bien que présentant une sécurité suffisante pour un procédé de contrôle rapide est inférieur à cet égard au procédé classique de numération sur plaques. Dans ses essais, il prend le résultat de la numération sur plaques comme base de comparaison. Or, il est bien connu, et nos résultats l'ont prouvé une fois de plus, que le procédé des plaques n'est pas d'une précision mathématique, bien s'en faut. Sachant cela, il est inadmissible de comparer simplement les deux procédés comme Richter l'a fait et de conclure que toutes les fois qu'il existe une différence, c'est le procédé Burri qui est en défaut. En se basant sur nos résultats, il paraît certain que les différences observées par RIcHTER entre le procédé des plaques et celui de BurRI proviennent tout aussi souvent de l'inexactitude du procédé qui a servi d'étalon que d'erreurs inhérentes au procédé essayé. Nous avons établi, en effet, que la dispersion des résultats de déterminations parallèles selon les deux procédés était du nême ordre de grandeur.

Il reste encore une question importante à discuter : Quel est le nombre maximum de colonies que l'on peut tolérer à la surface de la gélose en tubes sans que les résultats de la numération bactérienne en soient faussés. Le comité américain chargé d'établir des normes pour la numération bactérienne sur plaques a fixé à 300 le nombre maximum de colonies pour ce genre de culture. Richter (1. c.) va à 5 ou 600 par plaque. Si l'on voulait simplement comparer les surfaces ensemencées dans les deux procédés et en conclure au nombre de colonies admissible sur les tubes, on arriverait à 60 en prenant le 
chiffre de la commission américaine, et à 100-120 en prenant le chiffre de RICHTER, car la surface de la gélose inclinée est à peu près $1 / 5$ de celle d'une plaque de diamètre normal. Etant donné qu'il est probable qu'en fixant la limite à 300 , la commission américaine s'est laissée avant tout guider par des considérations d'ordre pratique. (le temps nécessaire pour dénombrer 300 colonies étant déjà très long pour un procédé destiné à la grande pratique et un nombre de colonies supérieur à 300 n'améliorant pas sensiblement la sûreté du procédé), on peut admettre qu'avec les nombres indiqués par RICHTER, la numération sur plaques donne encore des résultats sûrs. Il semblerait donc, qu'en se basant simplement sur la grandeur de la surface ensemencée, que 100 à 120 colonies par tube de gélose inclinée constituassent le maximum à fixer. Nous avons cependant tenu à trancherla question par l'expérience. Deux séries d'essais ont été faites dans le but de déterminer le maximum admissible de colonies. Ce sont les séries 4 et 5 , du tableau VII faites avec différentes dilutions d'une culture de str. lactis. En divisant le chiffre moyen obtenu dans chaque essai par la concentration du liquide employé pour l'ensemencement, on obtient un indice qui permet de décider à partir de quel nombre de colonies les résultats de ces essais sont trop bas. Nous donnons ci-après les résultats de ces essais :

TABLEAU VIII

NUMERATIONS COMPARATIVES PAR LE PROCEDE BURRI EN EMPLOYANT DES LIQUIDES D'ENSEMENCEMENT A DIFFÉRENTES GONGENTRATIONS.

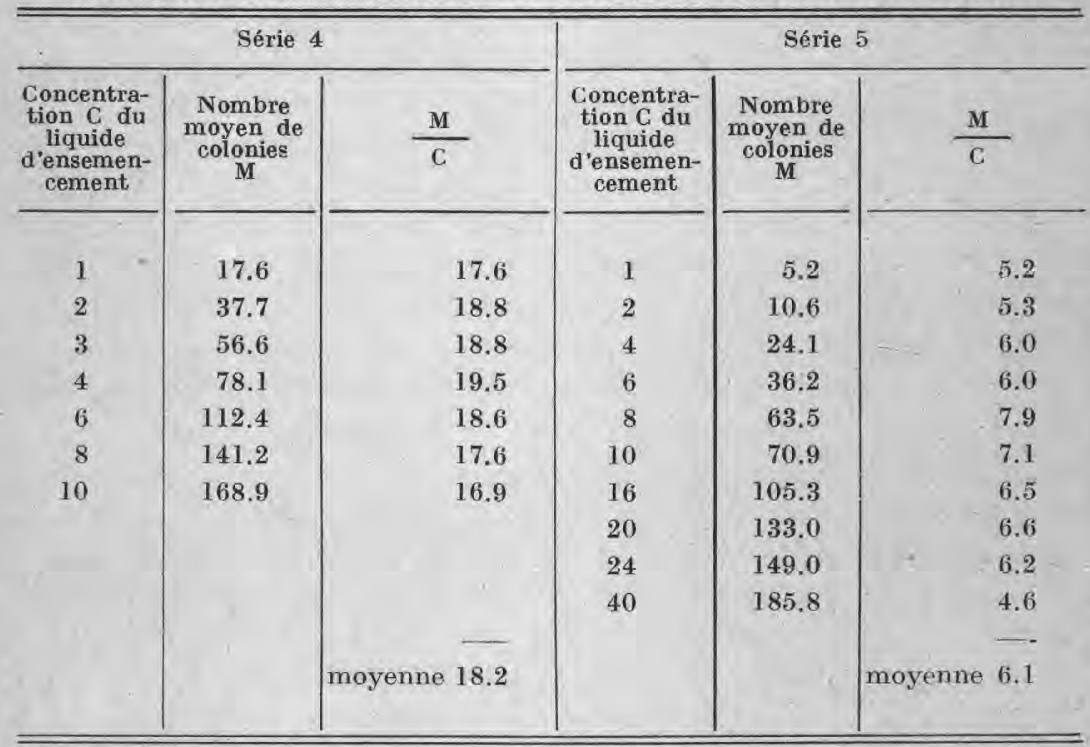

II ressort du tableau VIII que le nombre de bactéries que l'on trouve selon le procédé Burri diminue lorsque le nombre de colonies 
dépasse 150. Lorsque le nombre atteint 185 , on ne trouve que les $2 / 3$ environ du nombre qu'on est en droit d'attendre. Nous ne savons pas s'il faut attacher quelque importance au fait que dans les séries, ce sont les tubes contenant environ 60 à 80 colonies qui ont donné l'index le plus haut. De même, les tubes avec un nombre restreint de colonies ont donné dans les deux cas des indices relativement bas.

Quoi qu'il en soit, l'essai démontre que pour le str. lactis, le chiffre 150 constitue un maximum pour le nombre de colonies. Etant donné que ce microorganisme forme des colonies relativement petites, ce chiffre se trouve situé au-dessus du maximum admissible lorsqu'on travaille avec du lait. De nombreuses observations ont établi, en effet, que pour des bactéries produisant des colonies plus grandes, l'inhibition mutuelle se produit avec un nombre de colonies considérablement plus petit. C'est pour cette raison que nous proposons de fixer la limite supérieure du nombre de colonies par tube à 100. Il peut arriver exceptionnellement que même le nombre 100 est encore trop élevé. Cela dépend beaucoup des espèces bactériennes qui se trouvent dans le lait. Les tubes sont en effet ensemencés à la surface même de la gélose. Toutes les colonies sont done en surface et prennent davantage de place que lorsqu'elles sont situées en profondeur dans la gélose.

De ce qui précède, il s'ensulit que le procédé Burri peut être employé sans faire de dilutions pour du lait contenant jusqu'à 100.000 bactéries par $\mathrm{cm}^{3}$.

Il peut se pose encore la question : Des tubes présentant un nombre de colonies plus élevé que 100 permettent-ils, non pas la numération exacte qui est impossible, mais une estimation approximative de la teneur bactérienne du liquide ensemencé ? Cette estimation est faisable. Lorsqu'on examine les tubes après $24 \mathrm{~h}$, d'incubation on peut, si l'on dispose d'un peu d'expérience décider si un lait contient moins d'un million, jusqu'à 10 millions ou plus de 10 millions de bactéries par $\mathrm{cm}^{3}$.

VII. AVANTAGES ET INCONQÉNIENTS DES DIVERS PROCEDÉS DE NUMÉRATION BACTÉRIENNE UTILISÉS DANS LA PRATIQUE EN SUISSE.

Dans un prochain travail, nous prendrons position dans la controverse : essai de la réductase et numération bactérienne, en nous basant sur les résultats obtenus dans l'une des fromageries suisses d'expérimentation; et nous discuterons ici seulement les procédés de numération bactériënne par culture et à l'aide du microscope. La grande majorité des laits livrés en Suisse aux fromageries ou aux centres de ramassage ont une teneur en bactéries inférieure à 100.000 par $\mathrm{em}^{3}$. De ce fait, tous les procédés de numération à l'aide dumicroscope n'entrent pas sérieusement en ligne de compte pour le contrôle du lait livré par le paysan. Parmi les procédés, nous connaissons plus 
particulièrement celui de BREED ; il peut rendre d'excellents services lorsque le lait à examiner arrive en moyenne à une teneur microbienne de 300.000 au moins. Il pourrait également être très utile chez nous s'il s'agissait d'un simple contrôle et non de la détermination aussi exacte que possible de la teneur bactérienne. Le procédé Breed permet en effet de déterminer très rapidement d'une manière approximative si un lait contient par exemple moins de 100.000 ou plus de 300.000 bactéries par $\mathrm{cm}^{3}$. En outre, il peut donner des indications. sur la cause probable d'une teneur en bactéries trop élevée. En effet, on voit immédiatement au microscope si le lait contient beaucoup de leucocytes, signe d'une inflammation de la mamelle; on voit si les. bactéries se trouvent par gros paquets, signe d'un lavage insuffisant. des récipients; on peut voir finalement sile lait contient les diplocoques. caractéristiques du lait acide et l'on conclura à un refroidissement. insuffisant après la traite.

Etant donné qu'il s'agit pour nous non pas d'exclure le lait contenant par exemple plus de 100.000 bactéries au $\mathrm{cm}^{3}$, mais bien de trouver des différences entre des échantillons contenant 100.000 bactéries au $\mathrm{cm}^{3}$ au maximum, le procédé de BREed ne donne pas satisfaction.

Le procédé Burri et le procédé classique des plaques remplissent. les exigences que nous venons de formuler. Au point de vue pratique, ces procédés présentent de. grandes différences. Nous ne croyons pas que le procédé de numération bactérienne par plaques trouve jamais une application de quelque envergure, hors des Etats-Unis, où la routine le maintiendra encore un certain temps. Il est compliqué et nécessite un matériel encombrant et cher. En outre, il prend beaucoup de temps. Il est impossible d'effectuer une numération bactérienne par le procédé des plaques en dehors d'un laboratoire bien outillé, ear il faut de l'eau et tout un matériel stérilisé, des pipettes, des boîtes de Petri et encore un bain-marie pour fondre et refoidir la gélose, bref, du matériel encombrant. Il faut, en plus, beaucoup de temps. Pour le procédé Burri, tout ce dont on a besoin consiste en une anse calibrée, un verre d'eau, une lampe à alcool et des tubes de gélose inclinée. On peut même se passer de prélever des échantillons. Il suffit de tremper l'anse stérilisée dans le lait lorsque le fournisseur l'apporte. Le temps. pris pour effectuer un ensemencement est si court que l'on peut facilement suivre à l'arrivée des laits sans causer de retard dans le coulage.

Le procédé de BURRI est done bien le procédẻ de choix pour déterminer la teneur du lait en bactéries dans les conditions réalisées en Suisse. Sa rapidité et sa simplicité lui ouvriront aussi les portes des. usines laitières, où il permet de faire un contrôle serré à peu de frais.

VIII. RÉSUMÉ

Une étude détaillée du procédé Burri pour la numération bacté- 
rienne a été faite. Ce procédé se distingue par l'emploi d'une anse de platine calibrée pour mesurer la valeur d'un $\mathrm{mm}^{3}$ de lait et l'ensemencer à la surface de la gélose inclinée dans un tube. Le lait est réparti en imprimant un mouvement de zigzag à l'anse une fois qu'on a déposé le lait en un trait longitudinal à la surface de la gélose. Celle-ci doit être sèche et assez ferme pour résister à la friction de l'anse sans être éraillée. L'anse doit être rincée avant de la passer dans la flamme pour la stériliser. Les tủbes de gélose ensemencés sont placés debout. au thermostat à $30^{\circ} \mathrm{C}$. pour incubation.

On calibre l'anse en déterminant combien de colonies se développent dans des plaques ensemencées, d'une part, avec 10 anses de lait, et, d'autre part, avee $0 \mathrm{~cm}^{3} 01$ de lait mesuré à la pipette. Si l'anse retient la valeur de $1 \mathrm{~mm}^{3}$, le nombre de colonies trouvé dans les deux plaques sera le même. Nous avons trouvé de cette manière qu'en prenant du fil de platine iridié à $10 \%$ de $0 \mathrm{~mm} .38$ de diamètre, il fallait que l'anse ait un diamètre intérieur de $1 \mathrm{~mm} .50$ pour qu'elle transmette au milieu les bactéries de $0 \mathrm{~cm}^{3} 001$ de lait. Il est impossible d'effectuer directement la comparaison en mettant en regard les. résultats obtenus avec l'anse sur tubes et ceux obtenus sur plaques. ensemencées à la pipette, pour cette raison que les conditions du milieu sur tubes et la manière d'ensemencer y occasionnentle développement d'un nombre de colonies bien supérieur que sur plaques. Le procédé Burri donne des résultats avec une erreur probable de moins de $25 \%$ lorsqu'on compte entre 25 et 100 colonies à la surface de la gélose. Plus de 3.500 numérations ont prouvé que dans ces limites, ce procédé n'est en rien inférieur à celui des plaques quant à la sécurité.

Le peu de matériel employé, sa rapidité, sa simplicité et son degré de sécurité font du procédé Burri le procédé de choix pour la détermination de la teneur bactérienne du lait.

\section{BIBLIOGRAPHIE}

[1] Burri R. : Worlds Dairy Congress, London 1928, Report, p. 690-696; Le: Lait, 1929, 9 p.

[2] Conn H. W. : Public Health Reports of U.S. Publ. Health Service, vol. 30, p. 2349.

[3] Mrumer E. C. L. : J. of Lab. and Clin. Medicine St. Louis. IX, No. 6, 1924.

[4] SINGER \& HODER : Arch. f. Hyg., 1924.

[5] Richter K. : Molkereizeitung Hildesheim, 1931, Nr. 1, S. 5.

[6] Demont P. \& Dorner W. : Le Lart, 1931, p. 801.

[7] Dorner W. : Landw. Jahrbuch der Schweiz, 1930, p. 463.

[8] Bremp R. S. \& Dottrerer W. D. : Techn. Bull., Nr. 53, Agr. Exp. Sta., Geneva (N.Y.), 1916.

[9] MUdঞe C. S. \& LAWLer B. M. : Journ. of Bacteriology, 1928, 15, p. 207.

[10] Johannsen W.: Elemente der exakten Erblichleitslehre, G. Fischer, Jena, 1913. 